\title{
MEASURES OF GRAPHS ON THE REALS
}

\author{
SETH M. MALITZ
}

(Communicated by R. Daniel Mauldin)

\begin{abstract}
This paper studies measure properties of graphs with infinitely many vertices. Let $[0,1]$ denote the real unit interval, and $\mathscr{F}$ be the collection of bijections taking $[0,1]$ onto itself. Given a graph $G=\langle[0,1], E\rangle$ and $f \in \mathscr{F}$, define the $f$-representation of $G$ to be the set $E_{f}=\{\langle f(x), f(y)\rangle: x, y \in[0,1]$ and $\langle x, y\rangle \in E\}$. Let $\mu$ be 2-dimensional Lebesgue measure. Define the measure spectrum of $G$ to be the set $M(G)=\left\{m \in[0,1]: \exists f \in \mathscr{F}\right.$ with $E_{f}$ measurable and $\left.\mu E_{f}=m\right\}$. Our main result characterizes those subsets of reals that are the measure spectra of graphs.
\end{abstract}

\section{INTRODUCTION}

Consider an infinite graph $G=\langle[0,1], E\rangle$ where $[0,1]$ denotes the real unit interval. To each bijection of $[0,1]$ onto itself (i.e. each relabeling of the vertices of $G$ ) there corresponds a subset of the unit square which is essentially the adjacency matrix of $G$ under the specified labeling. This paper establishes some interesting relationships between the structure of a graph $G$ and measure properties of its corresponding family of adjacency matrices.

Our notation is as follows. We consider undirected graphs $G=\langle[0,1], E\rangle$ without loops or multiple edges. Let $\bar{G}$ denote the complement graph of $G$. Let $c$ denote the power of the continuum, $\mathbf{N}$ the natural numbers, and $\mathbf{N}^{+}$the positive natural numbers. For $s \in \mathbf{N}^{+} \cup\{\omega\}$, define $K_{s}(c)$ to be any complete $s$-partite graph with color classes of cardinality $c$, and $K_{c}$ to be any complete graph on $c$ vertices. Let $\mathscr{F}$ be the set of bijections of $[0,1]$ onto itself. Given a graph $G=\langle[0,1], E\rangle$ and an $f \in \mathscr{F}$, define the $f$-representation of $G$ to be the set $E_{f}=\{\langle f(x), f(y)\rangle: x, y \in[0,1]$ and $x, y \in E\}$. Take $\mu_{i}$ to be $i$-dimensional Lebesgue measure. Define the measure spectrum of a graph $G$ to be the set $M(G)=\left\{m \in[0,1]: \exists f \in \mathscr{F}\right.$ with $E_{f}$ measurable and $\left.\mu_{2} E_{f}=m\right\}$. Observe that $M(\bar{G})=1-M(G)=\{1-m: m \in M(G)\}$.

This paper provides solutions to the following three problems:

(1) Describe the subsets of $[0,1]$ that are the measure spectra of graphs and relate $M(G)$ to the structure of $G$.

Received by the editors September 23, 1986 and, in revised forms, December 3, 1987 and February 3, 1988.

1980 Mathematics Subject Classification (1985 Revision). Primary 28A05, 28A20, 05 C99. 
(2) Give necessary and sufficient conditions on a graph $G$ that insure $E_{f}$ is measurable for some $f \in \mathscr{F}$.

(3) Give necessary and sufficient conditions on a graph $G$ that insure $E_{f}$ is measurable for every $f \in \mathscr{F}$.

Most of the paper is devoted to question (1) because (2) is essentially answered in Blass [Bl], and (3) is relatively simple. As (1) is our main concern, let us get a feeling for what some typical measure spectra look like.

Example. If $G$ is a $K_{2}(c)$, then $M(G)=\left[0, \frac{1}{2}\right]$. To see this, let $A \subset[0,1]$ be the set of vertices in color class 1 , and $A^{c}$ the set of vertices in color class 2 .

$M(G) \supseteq\left[0, \frac{1}{2}\right]:$ For every $m \in\left[0, \frac{1}{2}\right]$, there is a $t_{m} \in\left[0, \frac{1}{2}\right]$ such that if $f_{m} \in \mathscr{F}$ maps $A$ onto $\left[0, t_{m}\right]$ and $A^{c}$ onto $\left(t_{m}, 1\right]$, then $\mu_{2} E_{f_{m}}=m$. To obtain a measure 0 representation, let $f_{0} \in \mathscr{F}$ map $A$ into the Cantor set.

$M(G) \subseteq\left[0, \frac{1}{2}\right]$ : Suppose $f \in \mathscr{F}$ and $E_{f}$ is measurable. Let $B=f[A]$. By Fubini's Theorem, $B$ is a measurable subset of $[0,1]$. Thus $\mu_{2} E_{f}=1-$ $\left(\mu_{1} B\right)^{2}-\left(1-\mu_{1} B\right)^{2} \leq \frac{1}{2}$.

In a similar way, one can prove that $M\left(K_{s}(c)\right)=[0,1-1 / s]$ for every $s \in \mathbf{N}^{+}$, and that $M\left(K_{\omega}(c)\right)=[0,1)$. It follows that if $G$ is $s$-colorable, then $G$ has no representation of measure $>1-1 / s$, and if $G$ is $\omega$-colorable, then $G$ has no representation of measure 1 . The disjoint union of a $K_{c}$ and a $\bar{K}_{c}$ is a graph with measure spectrum $[0,1]$.

\section{GRAPHS With PECULIAR MEASURE SPECTRA}

Proposition 2.1. There is a graph with empty measure spectrum.

Proof. The following construction is due to Sierpinski [Si]. Pick any bijection $g:[0,1] \rightarrow c$. Let $E$ consist of those pairs $\langle x, y\rangle \in[0,1]^{2}$ such that $g$ preserves the order of $x$ and $y$. The reader can verify that neither $G$ nor $\bar{G}$ contains a $K_{c}$. Thus, looking ahead to Theorem 5.1, $G$ has no measurable representation.

For $n \in \mathbf{N}^{+}$and $i, j \in\{0, \ldots, n-1\}$, define $S_{i j}^{n}$ to be the open square $(i / n,(i+1) / n) \times(j / n,(j+1) / n)$.

Proposition 2.2. For every $n \in \mathbf{N}^{+}$, there is a graph $G^{n}$ with measure spectrum $[0,1-1 / n] \cup\{1\}$.

Proof. Take the edge set $E$ defined in Proposition 2.1 and delete those pairs in the upper right and lower left quarters of $[0,1]^{2}$. Call this new set $E^{0}$. For each $n \in \mathbf{N}^{+}$define

$$
E^{n}=E^{0} \cup S_{11}^{2} \cup \bigcup_{\substack{0 \leq i, j \leq n-1 \\ i \neq j}} S_{i j}^{2 n}
$$

and $G^{n}=\left\langle[0,1], E^{n}\right\rangle$. Fix $f \in \mathscr{F}$ and set $A=f\left[\left[0, \frac{1}{2}\right]\right]$. Denote the representation $\left(E^{n}\right)_{f}$ by $E_{f}^{n}$. We claim that if $E_{f}^{n}$ is measurable, then $\mu_{1} A=0$ or 1. For contradiction, suppose not. There are two cases to consider. 
(i) $A$ is measurable and $0<\mu_{1} A<1$. Observe that for any $x \in\left[0, \frac{1}{2}\right]$, we have $\left|\left\{y \in\left[\frac{1}{2}, 1\right]:(x, y) \notin E^{0}\right\}\right|<c$, and for any $y \in\left[\frac{1}{2}, 1\right]$, we have $\left|\left\{x \in\left[0, \frac{1}{2}\right]:(x, y) \in E^{0}\right\}\right|<c$. Consequently, neither $E^{0} \cap\left(\left[0, \frac{1}{2}\right] \times\left[\frac{1}{2}, 1\right]\right)$ nor $\left(\left[0, \frac{1}{2}\right] \times\left[\frac{1}{2}, 1\right]\right)-E^{0}$ contains the Cartesian product of two sets $P, Q \subset[0,1]$ where $|P|=|Q|=c$. Hence neither $E_{f}^{0} \cap\left(A \times A^{c}\right)$ nor $\left(A \times A^{c}\right)-E_{f}^{0}$ contains the product of such sets.

If $E_{f}^{n}$ is measurable, then $E_{f}^{0} \cap\left(A \times A^{c}\right)$ is measurable. Since $0<\mu_{1} A<1$, either $E_{f}^{0} \cap\left(A \times A^{c}\right)$ or $\left(A \times A^{c}\right)-E_{f}^{0}$ has measure greater than zero, say $E_{f}^{0} \cap\left(A \times A^{c}\right)$ without loss of generality. By Theorem 3.3, $E_{f}^{0} \cap\left(A \times A^{c}\right)$ contains the Cartesian product of two nonempty perfect sets. But this is a contradiction as any nonempty perfect set has cardinality $c$. Thus $E_{f}^{n}$ is not measurable.

(ii) $A$ is nonmeasurable. Let $H_{1} \supset A$ be a $G_{\delta}$ set satisfying $\mu_{1} H_{1}=$ $\mu_{1}^{\text {outer }} A$, and $H_{2} \supset A^{c}$ be a $G_{\delta}$ set satisfying $\mu_{1} H_{2}=\mu_{1}^{\text {outer }} A^{c}$. Let $H_{3}=$ $H_{1} \cap H_{2}$. Clearly, $\mu_{1} H_{3}>0$ since $A$ is nonmeasurable. For $0 \leq k \leq$ $n-1$, define $B_{k}=f[(k / 2 n,(k+1) / 2 n)]$. Again, since $A$ is nonmeasurable, $\mu_{2}^{\text {outer }}\left(B_{k} \cap H_{3}\right)>0$ for some $k \in\{0,1, \ldots, n-1\}$, say $k=0$ without loss of generality. Let $H_{4} \supset B_{0} \cap H_{3}$ be a $G_{\delta}$ set satisfying $\mu_{2} H_{4}=\mu_{2}^{\text {outer }}\left(B_{0} \cap H_{3}\right)$. Notice $\mu_{2}^{\text {outer }}\left[E_{f}^{n} \cap\left(H_{4} \times H_{4}\right)\right]=\left(\mu_{1} H_{4}\right)^{2}$ because $\mu_{1}^{\text {outer }}\left(H_{4} \cap A^{c}\right)=\mu_{1} H_{4}$ and $A^{c} \times A^{c} \subset E_{f}^{n}$. However, $\mu_{2}^{\text {inner }}\left[E_{f}^{n} \cap\left(H_{4} \times H_{4}\right)\right]=0$ because $\mu_{1}^{\text {outer }}\left(H_{4} \cap B_{0}\right)=$ $\mu_{1} H_{4}$ and $B_{0} \times B_{0} \subset\left[E_{f}^{n}\right]^{c}$. Thus $E_{f}^{n}$ is nonmeasurable.

\section{LAYING THE GROUNDWORK}

Section 4 characterizes the subsets of $[0,1]$ that are the measure spectra of graphs. This characterization is obtained via the following ideas. Suppose $G$ has a representation $\mathscr{G}$ of measure $m \in(1-1 / r, 1-1 /(r+1))$ where $r \in \mathbf{N}^{+}$. We want to argue that $G$ has a representation of measure $m^{\prime}$ for every $m^{\prime} \in[0,1-1 /(r+1)]$. Let us first see that $G$ has a representation of measure $m^{\prime}$ for every $m^{\prime} \in[m, 1-1 /(r+1))$. Fix an arbitrarily small $\delta \in(0, m-(1-1 / r))$. By Theorem 3.1, it is possible to chop the unit square into a matrix of little squares so small that if we shade only those squares in which $\mathscr{G}$ has density greater than $1-\delta$, the set of shaded squares has measure within $\delta$ of $m$. By Turán's Theorem (see [Bo]) from extremal graph theory, there exists an $(r+1) \times(r+1)$ submatrix of little squares such that (1) the submatrix is symmetric about the line $y=x$, and (2) all squares of the submatrix not lying on the line $y=x$ are shaded. At this point, it is easy to show that $G$ has a representation of measure $m^{\prime}$ for every $m^{\prime} \in[m,(1-\delta)(1-1 /(r+1))]$. Since $\delta$ can be chosen arbitrarily small, $G$ has a representation of measure $m^{\prime}$ for every $m^{\prime} \in[m, 1-1 /(r+1))$. If we assume now that $G$ has no representation with measure greater than $1-1 /(r+1)$, then by Theorem 3.3, which asserts the existence of $c$-sized Cartesian squares in measurable sets satisfying certain 
properties, it can be shown that $G$ has a representation of measure $m^{\prime}$ for all $m^{\prime} \in[0,1-1 /(r+1)]$.

We now expand on these ideas formally. Given $n \in \mathbf{N}^{+}, i, j \in\{0, \ldots, n-$ $1\}$, and a measurable subset $F \subseteq[0,1]^{2}$, define $F_{i j}^{n}=F \cap S_{i j}^{n}$ where $S_{i j}^{n}$ is the open square $(i / n,(i+1) / n) \times(j / n,(j+1) / n)$. For $\varepsilon \in[0,1]$, say $F$ is $\varepsilon$-dense in $S_{i j}^{n}$ if $\mu_{2} F_{i j}^{n} / \mu_{2} S_{i j}^{n}>\varepsilon$. Let $F^{n, \varepsilon}$ be the collection of squares $S_{i j}^{n}$ in which $F$ is $\varepsilon$-dense.

Theorem 3.1. Let $F$ be a measurable subset of $[0,1]^{2}$, and $\varepsilon \in(0,1)$. Then

$$
\lim _{n \rightarrow \infty} \mu_{2} F^{n, \varepsilon}=\mu_{2} F .
$$

Proof. Let $O$ be an arbitrary open set containing $F$. Since $O$ is the countable union of open squares, it is easy to see

$$
\lim _{n \rightarrow \infty} \mu_{2} O^{n, 1}=\mu_{2} O \text {. }
$$

Now for all $n$,

$$
\mu_{2} O^{n, 1} \leq \mu_{2} O^{n, \varepsilon} \leq \mu_{2} O^{n, 1}+\frac{1}{n^{2}} \cdot \frac{\mu_{2}\left(O-O^{n, 1}\right)}{\varepsilon}
$$

which implies

$$
\lim _{n \rightarrow \infty} \mu_{2} O^{n, \varepsilon}=\mu_{2} O
$$

Thus

$$
\lim _{l \rightarrow \infty} \sup _{n>l} \mu_{2} F^{n, \varepsilon} \leq \lim _{l \rightarrow \infty} \sup _{n>l} \mu_{2} O^{n, \varepsilon}=\mu_{2} O .
$$

But since $O$ is arbitrary,

$$
\lim _{l \rightarrow \infty} \sup _{n>l} \mu_{2} F^{n, \varepsilon} \leq \mu_{2} F .
$$

In the other direction

$$
\lim _{l \rightarrow \infty} \inf _{n>l} \mu_{2} F^{n, \varepsilon}=1-\lim _{l \rightarrow \infty} \sup _{n>l} \mu_{2}\left(F^{c}\right)^{n, 1-\varepsilon} \geq 1-\mu_{2} F^{c}=\mu_{2} F .
$$

Lemma 3.2. Fix $n \in \mathbf{N}^{+}$. There is an $\varepsilon_{n} \in(0,1)$ such that for every measurable subset $D \subseteq[0,1]^{2}$, if $D$ is $\varepsilon_{n}$-dense in all the off-diagonal squares $S_{i j}^{n}$, there exist points $v_{i} \in(i / n,(i+1) / n), i=0, \ldots, n-1$, satisfying $\left\langle v_{i}, v_{j}\right\rangle \in D$ for all $0 \leq i, j \leq n-1$ with $i \neq j$.

Proof. Take $\varepsilon_{n}=1-1 / 2 n^{2}$ and choose the points $v_{i}$ in the appropriate intervals $(i / n,(i+1) / n)$ randomly, independently, with uniform distribution. For each fixed $i \neq j$, the probability that $\left\langle v_{i}, v_{j}\right\rangle$ fails to be in $D$ is at most $1 / 2 n^{2}$. So the probability that such a failure occurs for at least one of the $n(n-1)$ pairs is less than $1 / 2$. Thus some choice of $v_{i}$ 's satisfies the conclusion.

Given a measurable subset $F \subseteq[0,1]^{i}$, let $\phi(F)$ denote those points of $[0,1]^{i}$ at which $F$ has density 1 with respect to $\mu_{i}$. 
For $n \in \mathbf{N}$, let $\{0,1\}^{n}$ denote the set of binary strings of length $n$. If $\sigma$ and $\rho$ are two binary strings, let $\sigma \rho$ denote the concatination of $\sigma$ and $\rho$.

Mycielski [My] showed that if $E$ is a subset of the unit square of measure 1, then $E$ contains $P \times P$ for some nonempty perfect set $P \subset[0,1]$. Theorem 3.3, stated below, is an improvement of this result. Although it is stated only for dimension 2, the proof can be easily adapted to give an analogous result in all higher dimensions.

Theorem 3.3. Let $E$ be a measurable subset of $[0,1]^{2}$. If $\mu_{1}\{x \in[0,1]:\langle x, x\rangle \in$ $\phi(E)\}>0$, then there is a nonempty perfect set $P \subset[0,1]$ such that $P \times P-$ $\{\langle x, x\rangle: x \in P\} \subset E$.

Proof. First, we show that there is a closed subset $F \subseteq E$ such that $\mu_{1}\{x \in[0,1]:\langle x, x\rangle \in \phi(F)\}>0$. For each $i \in \mathbf{N}^{+}$, define $H_{i}$ to be the set of points in the plane whose distance from the line $y=x$ is greater than $1 / i$. Let $T_{i}=[0,1]^{2} \cap E^{c} \cap\left(H_{i+1}-H_{i}\right)$. Let $O_{i}$ be an open set in the plane that lies entirely within $H_{i+2}-H_{i-1}$, contains $T_{i}$, and satisfies $\mu_{2} O_{i}<\mu_{2} T_{i}+2^{-i}$. Now define $F$ to be $[0,1]^{2}-\bigcup_{i=1}^{\infty} O_{i}$. Obviously $F$ is a closed subset of $E$. Furthermore, any density point $\langle x, x\rangle$ of $E$ is also a density point of $F$. To see this, consider an $\alpha \times \alpha$ square $\mathscr{S}_{\alpha}$ rotated $45^{\circ}$ and centered at the point $\langle x, x\rangle \in \phi(E)$. For $\alpha \in(0,1)$, take $i_{\alpha}$ to be the largest integer $i$ such that $1 / i \geq \alpha / 2$. Assuming $\mathscr{S}_{\alpha}$ is contained in $[0,1]^{2}$, we have

$$
\mu_{2}\left(F^{c} \cap \mathscr{S}_{\alpha}\right)<\mu_{2}\left(E^{c} \cap \mathscr{S}_{\alpha}\right)+\sum_{j=i_{\alpha}}^{\infty} 2^{-j}=\mu_{2}\left(E^{c} \cap \mathscr{S}_{\alpha}\right)+2^{1-i_{\alpha}} \text {. }
$$

Since

obviously

$$
\lim _{\alpha \rightarrow 0} \frac{\mu_{2}\left(E^{c} \cap \mathscr{S}_{\alpha}\right)}{\alpha^{2}}=0 \text { and } \lim _{\alpha \rightarrow 0} \frac{2^{1-i_{\alpha}}}{\alpha^{2}}=0,
$$

$$
\lim _{\alpha \rightarrow 0} \frac{\mu_{2}\left(F^{c} \cap \mathscr{S}_{\alpha}\right)}{\alpha^{2}}=0 .
$$

Hence $\langle x, x\rangle$ is a density point of $F$. Thus $F$ is a closed subset of $E$ and $\mu_{1}\{x \in[0,1]:\langle x, x\rangle \in \phi(F)\}>0$.

Our task now is to prove the existence of a nonempty perfect set $P \subset[0,1]$ such that $P \times P-\{\langle x, x\rangle: x \in P\} \subset F$. Let $K_{1}=\{x \in[0,1]:\langle x, x\rangle \in \phi(F)\}$ and $K_{2}=\left\{x \in[0,1]: x \in \phi\left(K_{1}\right)\right\}$. For $j \in \mathbf{N}^{+}$, let $\varepsilon_{j}$ be defined as in Lemma 3.2. We want to construct a sequence of nested closed sets $J_{0} \supset J_{1} \supset J_{2} \supset \cdots$ whose intersection is the desired perfect set $P$. The sequence is defined inductively as follows.

For the base step, pick a point $z \in K_{2}$. Let

$$
\begin{gathered}
I=\left[z-a_{0}, z+a_{0}\right] \\
I_{0}^{\prime}=\left(z-a_{0}, z\right) ; \\
I_{1}^{\prime}=\left(z, z+a_{0}\right),
\end{gathered}
$$


and

$$
J_{0}=I
$$

where $a_{0}$ is chosen so small that

- $\mu_{1}\left(I_{\sigma}^{\prime} \cap K_{2}\right) / a_{0}$ is sufficiently close to 1 for each $\sigma \in\{0,1\}^{1}$ that the next inductive step can be performed;

- $\mu_{2}\left[\left(I_{\sigma}^{\prime} \times I_{\rho}^{\prime}\right) \cap F\right] / a_{0}^{2}>\varepsilon_{21}$ for all $\sigma, \rho \in\{0,1\}^{1}$ with $\sigma \neq \rho$.

Now let us proceed to the second step of the induction. By Lemma 3.2, there is a point $z_{\sigma} \in I_{\sigma}^{\prime} \cap K_{2}$ for each $\sigma \in\{0,1\}^{1}$ such that $\left\langle z_{0}, z_{1}\right\rangle,\left\langle z_{1}, z_{0}\right\rangle \in \phi(F)$. For all $\sigma \in\{0,1\}^{1}$, let

$$
\begin{gathered}
I_{\sigma}=\left[z_{\sigma}-a_{1}, z_{\sigma}+a_{1}\right] ; \\
I_{\sigma 0}^{\prime}=\left(z_{\sigma}-a_{1}, z_{\sigma}\right) ; \\
I_{\sigma 1}^{\prime}=\left(z_{\sigma}, z_{\sigma}+a_{1}\right),
\end{gathered}
$$

and

$$
J_{1}=\bigcup_{\sigma \in\{0,1\} 1} I_{\sigma},
$$

where $a_{1}$ is chosen so small that

- $I_{\sigma} \subset I_{\sigma}^{\prime}$ for all $\sigma \in\{0,1\}^{1}$;

- $\mu_{1}\left(I_{\sigma}^{\prime} \cap K_{2}\right) / a_{1}$ is sufficiently close to 1 for each $\sigma \in\{0,1\}^{2}$ that the next inductive step can be performed;

- $\mu_{2}\left[\left(I_{\sigma}^{\prime} \times I_{\rho}^{\prime}\right) \cap F\right] / a_{1}^{2}>\varepsilon_{2^{2}}$ for all $\sigma, \rho \in\{0,1\}^{2}$ with $\sigma \neq \rho$.

In the third step of the induction, we again use Lemma 3.2 to find points $z_{\sigma} \in I_{\sigma}^{\prime} \cap K_{2}$ for each $\sigma \in\{0,1\}^{2}$, so that $\left\langle z_{\sigma}, z_{\rho}\right\rangle \in \phi(F)$ for all $\sigma, \rho \in\{0,1\}^{2}$ with $\sigma \neq \rho$, and continue.

Repeating this procedure indefinitely, we obtain a nested sequence of closed sets $J_{0} \supset J_{1} \supset J_{2} \supset \cdots$. Let $P$ be the intersection of these $J_{i}$. Then $P$ is perfect and $P \times P-\{\langle x, x\rangle: x \in P\} \subset F$ since $F$ is closed.

Remark. The conclusion of Theorem 3.3 is not true if we assume only that $\{x \in[0,1]:\langle x, x\rangle \in \phi(E)\}$ has power the continuum.

Lemma 3.4. Suppose $G=\langle[0,1], E\rangle$ has a representation $\mathscr{G}$ of measure $m \in$ $(1-1 / r, 1-1 /(r+1))$ where $r \in \mathbf{N}^{+}$. Then $M(G) \supseteq[m, 1-1 /(r+1))$.

Proof. Fix an arbitrarily small positive $\delta<m-(1-1 / r)$. By Theorem 3.1, there is an integer $n_{1}$ such that $\left|\mu_{2} \mathscr{G}^{n, 1-\delta}-m\right|<\delta$ holds for all $n \geq n_{1}$. Notice that $\mathscr{G}^{n, 1-\delta}$ corresponds to the adjacency matrix of an $n$-vertex undirected graph $J_{n}$ by viewing each shaded square as a 1 , and each unshaded square as a 0 . As $n$ grows, the fraction of entries that are 1 in the adjacency matrix of $J_{n}$ approaches $m<1-(1 / r)$. Hence, by Turán's Theorem (see [Bo]) from extremal graph theory, there exists an $N \geq n_{1}$ such that for all $n \geq N$ the graph $J_{n}$ has a complete subgraph on $r+1$ vertices. In other words, there are integers $0 \leq w_{0}<w_{1}<\cdots<w_{r} \leq N-1$ such that $\mathscr{G}$ is $(1-\delta)$-dense in the 
square $\left(w_{i} / N,\left(w_{i}+1\right) / N\right) \times\left(w_{j} / N,\left(w_{j}+1\right) / N\right)$ for all $i, j \in\{0, \cdots, r\}$ with $i \neq j$.

At this point, it is clear that for every $m^{\prime} \in[m,(1-\delta)(1-1 /(r+1))], G$ has a representation of measure $m^{\prime}$. Since $\delta$ can be chosen arbitrarily small, $G$ has a representation of measure $m^{\prime}$ for every $m^{\prime} \in[m, 1-1 /(r+1))$.

Let $H^{n}$ be the set of all off-diagonal squares $S_{i j}^{n}$ where $i \neq j$. Let $\Gamma$ be the Cantor Set. Define an equivalence relation $\sim$ on the power set of $[0,1]^{2}$ as follows. Say $F \sim H$ if the symmetric set difference of $F$ and $H$ is contained in $(\Gamma \times[0,1]) \cup([0,1] \times \Gamma)$. Let $\langle F\rangle$ be the equivalence class of $F$ under $\sim$

For $n \in \mathbf{N}^{+}, D$ a subset of $[0,1]^{2}$, and $\mathscr{S}$ a square region in $[0,1]^{2}$ parallel with the coordinate axes, define ${ }^{o}[\mathscr{S} \cap D]$ to be the pure translation of $\mathscr{S} \cap D$ that takes the lower left corner of $\mathscr{S}$ to the origin.

Lemma 3.5. Suppose $G=\langle[0,1], E\rangle$ has a representation $\mathscr{G}$ of measure $m \in$ $(1-1 / r, 1-1 /(r+1)]$ where $r \in \mathbf{N}^{+}$. Suppose that for every $\nu>0$ there is an $m_{\nu} \in[1-1 /(r+1), 1-1 /(r+1)+\nu)$, such that $G$ has no representation of measure $m_{\nu}$. Then $G$ has a representation $\mathscr{G}^{\prime} \in\left\langle H^{r+1}\right\rangle$ and hence $M(G) \supseteq$ $[0,1-1 /(r+1)]$.

Proof. Proceed through the first paragraph in the proof of Lemma 3.4 with $0<\delta<m-(1-1 / r)$ and $1-\delta>\varepsilon_{r+1}$ where $\varepsilon_{r+1}$ is defined as in Lemma 3.2 .

Construct a new subset $\mathscr{G}^{*} \subset \mathscr{G}$ as follows. For each $a, b \in[0,1]$, define $K_{a, b}=\{x \in[0,1]:\langle x, x-a+b\rangle \in \phi(\mathscr{G})\}$. Let $\mathscr{G}^{*}=\left\{\langle a, b\rangle: a \in \phi\left(K_{a, b}\right)\right\} \cap \mathscr{G}$. By Fubini's Theorem and the Lebesgue Density Theorem, $\mathscr{G}^{*}$ is measurable and $\mu_{2}\left(\mathscr{G}-\mathscr{G}^{*}\right)=0$.

Applying Lemma 3.2 with $n=r+1$, choose points $v_{i} \in\left(w_{i} / N,\left(w_{i}+1\right) / N\right)$ where $i=0, \ldots, r$, so that $\left\langle v_{i}, v_{j}\right\rangle \in \mathscr{G}^{*}$ for all $0 \leq i, j \leq r$ with $i \neq j$. Pick $d$ sufficiently small that the intervals $I_{v_{i}}=\left[v_{i}-d, v_{i}+d\right]$ are all pairwise disjoint. Let

$$
A=\bigcap_{\substack{0 \leq i, j \leq r \\ i \neq j}}{ }^{o}\left[\left(I_{v_{t}} \times I_{v_{j}}\right) \cap \mathscr{G}^{*}\right] .
$$

Since all pairs $\left\langle v_{i}, v_{j}\right\rangle$ coincide in $A$, clearly $\mu_{1}\{x \in[0,2 d]:\langle x, x\rangle$ $\in \phi(A)\}>0$. Now it is not difficult to see that if $\langle x, x\rangle \in \phi(A)$, then ${ }^{o}\left[\left(I_{v_{i}} \times I_{v_{i}}\right) \cap \mathscr{G}\right]$ must have density 0 at $\langle x, x\rangle$ for every $i \in\{0, \ldots, r\}$. Otherwise, there is a $\nu>0$ such that $G$ has a representation of measure $m$ for every $m \in[1-1 /(r+1), 1-1 /(r+1)+\nu)$, which contradicts our hypothesis. So $\langle x, x\rangle \in \phi(A)$ implies ${ }^{o}\left[\left(I_{n_{1}} \times I_{v_{1}}\right) \cap \mathscr{G}^{c}\right]$ has density 1 at $\langle x, x\rangle$ for every $i \in\{0, \ldots, r\}$. Let

$$
B=A \cap \bigcap_{i=0}^{r}\left[\left(I_{v_{i}} \times I_{v_{i}}\right) \cap \mathscr{G}^{c}\right] .
$$

Since $\mu_{1}\{x \in[0,2 d]:\langle x, x\rangle \in \phi(B)\}=\mu_{1}\{x \in[0,2 d]:\langle x, x\rangle \in \phi(A)\}>0$, Theorem 3.3 tells us there is a nonempty perfect set $P \subset[0,2 d]$ such that 
$P \times P-\{\langle x, x\rangle: x \in P\} \subset B$. For each $i \in\{0, \ldots, r\}$, define $P_{i}=\left\{x+v_{i}-d: x \in\right.$ $P\}$. Observe that $P_{i} \times P_{j} \subset \mathscr{G}$ for all $i \neq j$, and $P_{i} \times P_{i} \subset \mathscr{G}^{c}$ for all $i$. Let $f \in \mathscr{F}$ satisfy

$$
f\left[[0,1]-\bigcup_{i=0}^{r} P_{i}\right]=\Gamma
$$

and

$$
f\left[P_{i}\right]=\left(\frac{i}{r+1}, \frac{i+1}{r+1}\right)-\Gamma .
$$

Then $E_{f} \in\left\langle H^{r+1}\right\rangle$, and hence $M(G) \supseteq[0,1-1 /(r+1)]$.

\section{THE MEASURE SPECTRA OF GRAPHS}

We are ready to prove our main theorem.

Theorem 4.1. For any graph $G=\langle[0,1], E\rangle, M(G)$ or $1-M(G)$ is one of the following subsets of reals:

(i) $\phi$;

(ii) $[0,1)$;

(iii) $[0,1]$;

(iv) $\left[0,1-\frac{1}{s}\right], \quad s \in \mathbf{N}^{+}$;

(v) $\left[0,1-\frac{1}{s}\right] \cup\{1\}, \quad s \in \mathbf{N}^{+}$.

Conversely, each of the above subsets of reals is the measure spectrum of some graph. If $M(G)$ is of type (iv) or (v), then $G$ has a representation $\mathscr{G} \in\left\langle H^{s}\right\rangle$. Proof. Suppose that $M(G)$ is not one of the following subsets of reals: $\phi$, $[0,1],(0,1],[0,1),\{0\},\{1\}$ or $\{0,1\}$. Looking ahead to Theorem $5.1, M(G)$ $\neq(0,1)$. Hence there are $m_{0}, m_{1} \in(0,1)$ where $m_{0} \in M(G)$ and $m_{1} \notin$ $M(G)$.

Let $\alpha=\inf [(0,1)-M(G)]$ and $\beta=\inf [M(G) \cap(0,1)]$. Notice $\alpha$ and $\beta$ are well-defined. We have two cases to consider:

(i) $\alpha>0$. Since $M(G) \supseteq(0, \alpha) \neq \phi$, Lemma 3.4 says $\alpha=1-1 / s$ for some $s \geq 2$. By Lemma 3.5, $M(G) \supseteq[0,1-1 / s] \supseteq\left[0, \frac{1}{2}\right]$. We claim that $G$ has no representation of measure $m_{2}$ where $\frac{1}{2} \leq \alpha<m_{2}<1$. Suppose for contradiction that it did. Applying Lemma 3.4 to $\bar{G}$ gives $M(\bar{G}) \supseteq\left[1-m_{2}, \frac{1}{2}\right)$ which implies $M(G) \supseteq\left(\frac{1}{2}, m_{2}\right]$. Since we already know $M(G) \supseteq\left[0, \frac{1}{2}\right]$, the last statement implies $M(G) \supseteq\left[0, m_{2}\right]$. Hence $\alpha \geq m_{2}$, a contradiction.

(ii) $\alpha=0$. If $\alpha=0$, then $\beta>0$ by Lemma 3.4. Since $1-\beta=\sup [(0,1) \cap$ $M(\bar{G})$ ], Lemma 3.4 says $1-\beta=1-1 / s$ for some $s \geq 2$. So $(1-1 / s, 1) \subset$ $(0,1)-M(\bar{G})$. By Lemma 3.5, $M(\bar{G}) \supseteq[0,1-1 / s]$.

At the end of the introduction, we gave examples of graphs with measure spectra of type (ii), (iii) and (iv). Proposition 2.1 yielded a graph with empty measure spectrum, and Proposition 2.2 produced a graph with measure spectrum $[0,1-1 / s] \cup\{1\}$ for each $s \in \mathbf{N}^{+}$. 
Finally, Lemma 3.5 implies that if $M(G)$ is of type (iv) or (v), then $G$ has a representation $\mathscr{G} \in\left\langle H^{s}\right\rangle$.

Remark. If $M(G)$ is of type (iv) or (v), then clearly $s$ must be the largest integer $n$ for which $G$ contains $K_{n}(c)$ as induced subgraph.

Remark. If we define $\mathscr{F}^{\prime}=\{f \in \mathscr{F}: f$ measurable $\}$ and $M^{\prime}(G)=\{m \in$ $[0,1]: \exists f \in \mathscr{F}^{\prime}$ such that $E_{f}$ is measurable and $\left.\mu_{2} E_{f}=m\right\}$, then Theorem 4.1 still holds for $M^{\prime}(G)$. In general however, $M^{\prime}(G) \neq M(G)$ even if $E$ is a measurable subset of $[0,1]^{2}$.

Proposition 4.2. If $G=\langle[0,1], E\rangle$ is a graph for which $M(G)=[0,1)$, then $G$ has a representation $\mathscr{G}^{n} \in\left\langle H^{n}\right\rangle$ for every $n \in \mathbf{N}^{+}$.

Proof. It is not too difficult to construct an argument using ideas in the proof of Lemma 3.5, the proof of Theorem 5.1, and the fact that $G$ contains no $K_{c}$.

Remark. If $M(G)=[0,1)$, then $G$ must contain a $K_{s}(c)$ as an induced subgraph for every $s \in \mathbf{N}^{+}$.

\section{INSURING THAT SOME REPRESENTATION IS MEASURABLE}

The next theorem states conditions under which $G$ has a measurable representation.

Theorem 5.1. The following are equivalent:

(i) $G=\langle[0,1], E\rangle$ has a measurable representation.

(ii) $G$ or $\bar{G}$ contains $a K_{c}$.

(iii) $G$ has a representation of measure 0 or 1 .

The proof is a consequence of the following result due to Galvin [Ga].

Theorem $5.2[\mathrm{Ga}]$. Let $P \subseteq[0,1]$ be perfect and nonempty. Let $B \subseteq[0,1]^{2}$ be a Borel set symmetric about the line $y=x$. Then there is a nonempty perfect set $P^{\prime} \subseteq P$ such that $P^{\prime} \times P^{\prime}-\left\{\langle x, x\rangle: x \in P^{\prime}\right\} \subseteq B$ or $B^{c}$.

Blass [Bl, p. 271] supplies the following verification of Theorem 5.1. The only nontrivial direction, of course, is (i) $\Rightarrow$ (ii): Suppose, without loss of generality, $E$ is measurable. Let $F \subseteq E$ be an $F_{\sigma}$ set such that $\mu_{2}(E-F)=0$ and $H \supseteq E$ be a $G_{\delta}$ set such that $\mu_{2}(H-E)=0$. Let $V=F \cup H^{c}$ and observe $\mu_{2} V=1$. By Theorem 3.3 (or the result of Mycielski [My]), there is a nonempty perfect $P \subset[0,1]$ satisfying $P \times P-\{\langle x, x\rangle \mid x \in P\} \subset V$. By Theorem 5.2, there is a nonempty perfect $P^{\prime} \subset P$ such that $P^{\prime} \times P^{\prime}-\left\{\langle x, x\rangle: x \in P^{\prime}\right\} \subset F$ or $F^{c}$, which implies $P^{\prime} \times P^{\prime}-\left\{\langle x, x\rangle: x \in P^{\prime}\right\} \subset E$ or $E^{c}$.

The proof of Theorem 5.1 has another interesting consequence. Let $X$ be any subset of $[0,1]$ and define $X^{*}=\{y-x \mid x, y \in X$ and $y>x\}$.

Proposition 5.3. For every measurable $A \subseteq[0,1]$ there is a nonempty perfect set $P \subseteq[0,1]$ such that $P^{*} \subseteq A$ or $P^{*} \subseteq A^{c}$. 
Proof. Define $E=\{\langle u, v\rangle \mid u, v \in[0,1]$ and $|u-v| \in A\} . E$ is easily seen to be a measurable subset of $[0,1]^{2}$, symmetric about the line $y=x$. The proof of Theorem 5.1 says there is a perfect set $P \subset[0,1]$ such that $P \times P-\{\langle x, x\rangle \mid x \in$ $P\} \subset E$ or $P \times P-\{\langle x, x\rangle \mid x \in P\} \subset E^{c}$. In other words, $P^{*} \subset A$ or $P^{*} \subset A^{c}$.

Remark. Carlson [Ca] has constructed a nonmeasurable set $A \subset[0,1]$ such that for every $X \subseteq[0,1]$ of cardinality $c, X^{*} \cap A \neq \varnothing$ and $X^{*} \cap A^{c} \neq \varnothing$.

\section{INSURING THAT EVERY REPRESENTATION IS MEASURABLE}

The next theorem states conditions under which every representation of $G$ is measurable. First we need a lemma. The proof is essentially that of Theorem 3.1, p. 375 in $[\mathrm{Ba}]$.

Lemma 6.1. Let $G$ be the graph with vertex set $[0,1]$ whose only edges are $\left\{x, x+\frac{1}{3}\right\}$ where $x$ ranges over $\left[0, \frac{1}{3}\right]$. Then $G$ has a representation of outer measure 1 .

Proof. Let $F_{y}(\gamma<c)$ enumerate all closed subsets of $\left\{\langle x, y\rangle \in[0,1]^{2} \mid x<y\right\}$ having positive measure. By induction on $\beta<c$, pick distinct $p_{\beta}, q_{\beta}, r_{\beta}$ so that

- $p_{\beta}, q_{\beta}, r_{\beta} \notin \bigcup_{\gamma<\beta}\left\{p_{\gamma}, q_{\gamma}, r_{\gamma}\right\}$ and

- $\left\langle p_{\beta}, q_{\beta}\right\rangle \in F_{\beta}$.

Note that the choice of $p_{\beta}, q_{\beta}, r_{\beta}$ is possible by Fubini's Theorem. Let $A=\bigcup_{\beta<c}\left\{p_{\beta}, q_{\beta}\right\}$ and $g:\left[0, \frac{1}{3}\right] \rightarrow c$ be $1-1$ and onto. Observe that $A^{c}$ has cardinality $c$. Pick $f \in \mathscr{F}$ such that $f\left[\left[\frac{2}{3}, 1\right]\right]=A^{c}$, and $\left\langle f(x), f\left(x+\frac{1}{3}\right)\right\rangle=$ $\left\langle p_{g(x)}, q_{g(x)}\right\rangle$ for every $x \in\left[0, \frac{1}{3}\right]$. Then the complement of $E_{f}$ contains no closed set of positive measure and hence has inner measure 0.

A subset $X \subseteq[0,1]$ is a vertex cover for the graph $G=\langle[0,1], E\rangle$ if for every edge $\langle u, v\rangle \in E$ either $u \in X$ or $v \in X$ or both.

Theorem 6.2. The following are equivalent:

(i) Every representation of $G=\langle[0,1], E\rangle$ is measurable.

(ii) $G$ or $\bar{G}$ has a vertex cover of cardinality smaller than every subset of $[0,1]$ of outer measure 1 .

(iii) Every representation of $G$ has measure 0 or every representation of $G$ has measure 1.

Proof. (i) $\Rightarrow$ (ii). Suppose neither $G$ nor $\bar{G}$ has a vertex cover of cardinality $\gamma<c$. Then $G$ and $\bar{G}$ both contain a set of $c$ disjoint edges. Therefore, by Lemma $6.1, G$ and $\bar{G}$ both have representations with outer measure 1 . Suppose, for contradiction, that every representation of $G$ is measurable. Then $G$ and $\bar{G}$ both have measurable representations of measure 1 . Therefore, by Theorem 3.3, there are disjoint subsets $A, B \subset[0,1]$ of cardinality $c$, such that $A \times A-\{\langle x, x\rangle: x \in A\} \subset E$ and $B \times B-\{\langle x, x\rangle: x \in B\} \subset E^{c}$. Let 
$D=[0,1]-(A \cup B)$. Pick $f \in \mathscr{F}$ such that $f[D]$ is a set of measure 0 , $f[A]=\mathscr{V}-f[D]$ and $f[B]=\mathscr{V}^{c}-f[D]$, where $\mathscr{V} \subset[0,1]$ is a nonmeasurable set of cardinality $c$ whose complement also has cardinality $c$. Then $E_{f}$ is nonmeasurable.

(ii) $\Rightarrow$ (iii) Suppose $G=\langle[0,1], E\rangle$ has a vertex cover $C$ of cardinality smaller than any subset of $[0,1]$ of outer measure 1 . Then obviously $C$ has measure 0 . For any vertex $v$ of $G$, if $v \notin C$ then $\{w:\langle v, w\rangle \in E\} \subseteq C$. Thus by Fubini's Theorem, every representation of $G$ must have measure 0 .

\section{OPEN QUESTIONS}

It would be interesting to obtain analogues of Theorem 4.1 for directed graphs, hypergraphs, other measures besides Lebesgue measure, and graphs of higher cardinality. With regard to hypergraphs, there is a conjecture known as Turán's Conjecture which is the hypergraph analogue to Turán's Theorem about graphs. Unfortunately this conjecture has eluded proof for over forty years (see Kalai [Ka]). Thus, we suspect a hypergraph version of Theorem 4.1 will be difficult to establish. The situation for directed graphs looks a little more promising.

\section{ACKNOWLEDGMENTS}

I would like to thank Jerome Malitz for suggesting the theme of this paper, and for his encouragement, enthusiasm and many helpful discussions. My appreciation also goes to Tim Carlson, Rich Laver, Jan Mycielski and Monty McGovern for all their assistance. Finally, I would like to thank an anonymous referee for dramatically simplifying an earlier proof of Lemma 3.2, and for a number of suggestions that improved the organization of the paper.

\section{REFERENCES}

[Ba] J. Barwise, ed., Handbook of mathematical logic, North-Holland, 1977.

[BI] A. Blass, A partition theorem for perfect sets, Proc. Amer. Math. Soc. 82 (1981), 271-277.

[Bo] B. Bollabas, Graph theory-an introductory course, Springer-Verlag New York Inc., 1979, p. 72.

[Ca] T. Carlson, personal communication.

[Ga] F. Galvin, Partition theorems for the real line, Notices Amer. Math. Soc. 15 (1968), 660; Erratum 16 (1969), 1095.

[Ha] F. Harary, Graph theory, Addison-Wesley Publishing Co., 1972.

[Ka] G. Kalai, A new approach to Turán's Conjecture, Graphs and Combinatorics 1 1986, 107-109.

[My] J. Mycielski, Algebraic independence and measure, Fund. Math. 61 (1967), 165-169.

[Si] W. Sierpinski, Sur un probleme de la theorie des relations, Ann. Scuola Norm. Sup. Pisca 2 (1933), 285-287.

Ashdown House, Room 615A, 305 Memorial Drive, Cambridge, Massachusetts 02139

Current address: Department of Computer and Information Sciences, University of Massachusetts, Amherst, Massachusetts 01003 